Anales de Geografía de la Universidad Complutense ISSN: 0211-9803

http://dx.doi.org/10.5209/AGUC.57731

\title{
Estimación de bajas temperaturas en la Isla de Tenerife y valoración de resultados para el cultivo de mango, guayaba y noni en agricultura ecológica
}

\author{
Desiderio Alberto Francisco Bethencourt ${ }^{1}$ \\ Recibido: 12 de julio del 2016 / Enviado a evaluar: 19 de julio del 2016 / Aceptado: 16 de diciembre del 2016
}

\begin{abstract}
Resumen. En Tenerife la superficie de frutales tropicales y subtropicales en agricultura ecológica ha aumentado significativamente en los últimos años, cultivándose principalmente en zona de costa donde predominan los aridisoles. No obstante, el clima es un factor a considerar tomando en cuenta que episodios de olas de frío pueden ocurrir y ocasionar daños en árboles jóvenes. Se pretende identificar áreas potenciales para el cultivo de mango, guayaba y noni con un manejo ecológico como alternativa de producción en fincas abandonadas. Para esto planteamos que en relieves montañosos la interacción de los factores altitud y temperatura, pueden relacionarse con la pendiente de la ladera para delimitar áreas de cultivo. El trabajo se realiza con datos de altitud y temperatura mínima absoluta diaria, obteniéndose isotermas mediante modelo matemático que considera el gradiente térmico altitudinal. Las isotermas se interpolan por kriging en un modelo de elevación digital y se reclasifican mediante análisis multicriterio, tomando en cuenta la pendiente más favorable del terreno. Los mapas resultantes muestran la distribución de bajas temperaturas (frío y heladas), y áreas con potencial actual y futuro para los cultivos estudiados.
\end{abstract}

Palabras clave: Gradiente térmico altitudinal; modelo de elevación digital; kriging; árboles jóvenes.

\section{[en] Estimation of low temperatures on the island of Tenerife and impact assessment for growing mango, guava and noni in organic farming}

\begin{abstract}
Tenerife surface in tropical and subtropical fruit in organic farming has increased significantly in recent years, mainly cultivated in coastal area where Aridisols predominate. However, the weather is a factor considering that episodes of cold waves can occur and damage young trees. It is intended to identify potential for organic growing mango, guava and noni as an alternative farm production in abandoned areas. For this we propose that in mountainous terrain interaction of altitude and temperature factors may be related to the slope of the hillside to delimit cultivation areas. The work is done with altitude data and daily absolute minimum temperature, isotherms obtained by mathematical model that
\end{abstract}

1 Asociación Cereales de Tenerife (ACETE). Tenerife, Canarias.

E-mail: desideriof@gmail.es 
considers the altitudinal thermal gradient. The isotherms are interpolated by kriging on a digital elevation model and reclassified by multicriteria analysis, taking into account the more favorable terrain slope. The resulting maps show the distribution of low temperatures (cold and frost), and appropriate areas with current and future potential for crops studied.

Key words: Altitudinal thermal gradient; digital elevation model; kriging; young trees.

\section{[fr] Estimation des basses températures sur l'île de Tenerife et de l'évaluation de l'impact pour la croissance de la mangue, la goyave et noni en agriculture biologique}

Résumé. Surface Tenerife dans les fruits tropicaux et subtropicaux en agriculture biologique a considérablement augmenté au cours des dernières années, principalement cultivée dans la zone côtière où les Aridisols prédominent. Cependant, le temps est un facteur considérant que les épisodes de vagues de froid peuvent se produire et endommager les jeunes arbres. Il est destiné à identifier le potentiel de bio mangue croissante, la goyave et noni comme une production agricole de remplacement dans les zones abandonnées. Pour cela, nous proposons que dans l'interaction du relief montagneux de facteurs d'altitude et de température peut être liée à la pente de la colline pour délimiter les zones de culture. Le travail se fait avec les données d'altitude et la température minimale absolue quotidien, isothermes obtenus par modèle mathématique qui tient compte du gradient thermique altitudinal. Les isothermes sont interpolées par krigeage sur un modèle numérique d'élévation et reclassées par une analyse multicritères, en tenant compte de la pente du terrain plus favorable. Les cartes obtenues montrent la répartition des basses températures (froid et de gel), et les zones appropriées avec le potentiel actuel et futur pour les cultures étudiées.

Mots clés: gradient thermique altitudinal, modèle numérique d'élévation, krigeage, gaules.

Cómo citar. Francisco Bethencourt, D.A. (2017): Estimación de bajas temperaturaes en la Isla de Tenerife y valoración de resultados para el cultivo de mango, guayaba y noni en agricultura ecológica. Anales de Geografía de la Universidad Complutense, 37(2), 399-414.

Sumario. 1. Introducción. 2. Materiales y métodos. 2.1. Información SIG. 2.2. Los datos y su tratamiento. 3. Resultados y discusión. 3.1. Datos climátológicos. 3.2. Modelo de elevación digital. 3.3. Gradiente térmico altitudinal. 3.4. Confiabilidad del ráster Tma. 3.5. Mapa de distribución espacial de bajas temperaturas. 3.6. Mapa de áreas potenciales para nuevas plantaciones. 4. Conclusones. 5. Bibliografía.

\section{Introducción}

El abandono de las tierras agrícolas tiene consecuencias negativas para el medio rural (por ej. un paisaje desolado) y para el medio ambiente (por ej. propagación de incendios) de la isla de Tenerife.

Según datos del mapa de cultivos de la isla, unas 24543 ha se encuentran sin cultivar debido tanto al abandono reciente, como al abandono prolongado (LópezManzanares y Machín, 2012). Por otra parte, el 6,8\% de la superficie cultivada de Tenerife se dedica a los cereales (trigo, cebada, centeno, avena y triticale) y los agricultores de cereales buscan alternativas para diversificar sus explotaciones. En este sentido, el cultivo de árboles frutales tropicales y subtropicales se plantea como 
una opción válida tanto para la recuperación de fincas abandonadas, como en la diversificación de explotaciones agrarias.

La agricultura ecológica es una alternativa para cultivar un suelo productivo con el fin de obtener un producto de calidad (Castillo, 1999). A nivel de Canarias, la superficie cultivada en agricultura ecológica de frutales tropicales y subtropicales ha pasado de 62 a 88,4 ha en el período 2012-2013 (incremento de 68,7\%). Este es un dato significativo si se compara con el cultivo de plátano ecológico (cultivo tradicional y de gran demanda) que solo ha crecido un $31,3 \%$ en el mismo período (MAGRAMA, 2013 y MAGRAMA, 2014).

Los frutales subtropicales constituyen un subsector reciente en la producción agrícola de Tenerife. El $65 \%$ de la superficie cultivada se asienta en zonas de costa, entre 15 y $400 \mathrm{msnm}$, mientras que el 35\% restante llega hasta los $1065 \mathrm{msnm}$ en las medianías de la isla. En cuanto a suelos, una superficie que representa el 63,5\% (129,5 ha) de dichos frutales, se cultiva sobre aridisoles.

Entre los factores que se deben considerar para el cultivo de un árbol frutal tropical o subtropical en agricultura ecológica se encuentran: las condiciones climáticas, el tipo de suelo, la disponibilidad y calidad de agua de riego y la incidencia de agentes fitopatógenos. En este sentido, el mango (Mangifera indica L.), la guayaba (Psidium guajava L.) y el noni (Morinda citrifolia L.) son cultivos tropicales que pueden adaptarse a diferentes clases de suelos, siempre que estos sean profundos y con buen drenaje. Sin embargo, al considerar el clima para la viabilidad de una plantación de frutales, se debe tomar en cuenta la temperatura como un factor que puede condicionar la planificación de dichos cultivos en Tenerife. Es por ello que en adelante se hace referencia principalmente a este factor.

La temperatura media se debe conocer, sobre todo en los meses más críticos para el desarrollo de un árbol frutal (invierno y primavera) donde las bajas temperaturas pueden afectar su crecimiento y floración. Para Paul et al. (2011), la vulnerabilidad de los frutales al cambio de temperatura tiene su impacto en la eficiencia de la polinización y la producción de frutos. Por ello, en Tenerife se recomienda una temperatura media anual para el cultivo de mango de $24-27^{\circ} \mathrm{C}$, con mínima de $18^{\circ} \mathrm{C}$ y máxima de $35^{\circ} \mathrm{C}$ (Galán, 1993) y en la guayaba de media $23-28^{\circ} \mathrm{C}$, con 18 y $30^{\circ} \mathrm{C}$ de mínima y máxima respectivamente (Fernández y Hernández, 2013), mientras que para el noni no se han publicado datos. Sin embargo, tomando de referencia datos de Hawai (USA), se ha recomendado para el noni una temperatura media anual de 25$30^{\circ} \mathrm{C}$, con mínima de $20^{\circ} \mathrm{C}$ y máxima de $35^{\circ} \mathrm{C}$ (Nelson, 2003).

Por otra parte, entre las amenazas de origen climático que pueden suceder en Canarias se encuentran las "olas de frío" (Dorta, 2007). Una ola de frío, es un episodio de temperaturas anormalmente bajas y que se mantiene varios días. De acuerdo con Cardós et al. (2006), las olas de frío que han llegado a Canarias han tenido una persistencia de 3-4 días y una temperatura media de $14,3^{\circ} \mathrm{C}$. Estas se producen por la invasión de una masa de aire muy frío del ártico, que es desplazada desde la península ibérica a las islas por el Anticiclón de las Azores. 
Si la temperatura ambiente desciende por debajo de $0^{\circ} \mathrm{C}$ y se produce un daño irreversible en los tejidos de la planta, se llama helada (desde el punto de vista agronómico). Un método pasivo para mitigar el posible daño que causen las heladas, consiste en seleccionar el lugar donde se va a plantar el frutal.

En el futuro según Easterling et al. (2016), los fenómenos meteorológicos extremos como olas de frío, no solo serán numerosos sino también más intensos. Hasta la fecha, en Canarias no se han realizado estudios que tomen en cuenta estos episodios de bajas temperaturas por suceder, para el establecimiento de nuevas plantaciones de frutales tropicales y subtropicales.

A su vez el frío, entendiéndose este como temperaturas entre 0 y $15^{\circ} \mathrm{C}$, puede causar daños de consideración en los árboles jóvenes, si la temperatura se mantiene por debajo del umbral crítico durante varias horas consecutivas. El umbral crítico es la temperatura más baja que es segura. En tal sentido, Francisco-Bethencourt (2015) ha identificado y descrito la sintomatología de daños por frío que le ocurre a los plantones de mango, guayaba y noni cuando se someten a una temperatura inferior a $12^{\circ} \mathrm{C}$ (umbral crítico).

El presente trabajo postula que en relieves montañosos la interacción de los factores altitud y temperatura, pueden relacionarse con la pendiente de la ladera para delimitar áreas potenciales de cultivo. Por ello se ha realizado este estudio a objeto de (i) fijar los límites en la distribución espacial de bajas temperaturas en la isla de Tenerife e (ii) identificar, tomando en cuenta esas variaciones, áreas potenciales para el cultivo de mango, guayaba y noni con un manejo ecológico.

\section{Materiales y métodos}

\subsection{Información SIG}

El uso de los Sistemas de Información Geográfica (SIG) como herramienta capaz de gestionar y analizar la información espacial, ha propiciado trabajos de investigación con el fin de elaborar y validar mapas de variables, tales como, fertilidad del suelo (Henríquez et al. 2013) o precipitación (Izquierdo y Márquez, 2007). Para la elaboración de estos estudios se emplea un software informático.

En el presente estudio se ha empleado gvSIG. Este software (Open Source), permite recopilar, organizar, administrar, analizar, compartir y distribuir la información geográfica. Por otra parte, se tomaron datos de variables continuas (temperatura y altitud) es decir, se encuentran distribuidas de forma continua en cualquier lugar y por tanto cualquier pixel tendrá un valor de dicha variable.

El pixel es el elemento más pequeño al que puede asignarse de forma independiente el valor de una variable y conformar un ráster. El ráster es un modelo de datos que se obtiene del SIG, en el cual las variables son representadas usando pixeles (celdas) cuadrados, ordenados conformando una grilla (cuadrícula) regular. 
Los modelos digitales del terreno, ráster, son el tipo de dato SIG más habitual. Cuando la variable es elevación, se denomina Modelo de Elevación Digital (DEM).

La capa base para este estudio es un DEM. Para generarlo se toman los datos de curvas de nivel de la isla de Tenerife (distanciadas $25 \mathrm{~m}$ ) y se emplean herramientas de geoprocesamiento del software utilizado.

\subsection{Los datos y su tratamiento}

La temperatura mínima absoluta diaria (Tma), es una variable climática cuyo comportamiento depende de diversos factores, entre otros, la altitud. Para este estudio se han recopilado datos de Tma de 57 estaciones meteorológicas pertenecientes a la red del Cabildo Insular de Tenerife. Las estaciones se encuentran situadas a diferentes altitudes.

Los datos de Tma consultados son del año 2014, siendo el comportamiento térmico registrado normal si se compara con el período de referencia (1971-2000). Se analizan los valores de las medias mensuales para determinar el mes más frío y se observa que corresponde a febrero. Luego, con los datos diarios de ese mes, en cada estación se obtienen los tres valores más bajos y con ellos se hace una media ponderada que es el dato que representa a cada estación meteorológica.

Las estaciones meteorológicas se encuentran georreferenciadas y esto permite emplear herramientas SIG para analizar el comportamiento espacial de los datos de las variables altitud y Tma con respecto a las vertientes (norte y sur) de la isla. Dichas vertientes se representan mediante el cruce de las líneas que dibujan las dorsales Teno-Pico Teide y Anaga-Pico Teide (Figura 2). La estadística descriptiva para los datos de cada variable son: rango (valores mínimo y máximo), número de observaciones (n), media $(\overline{\mathrm{X}})$ y desviación estándar (s).

A continuación se explican los pasos seguidos para la obtención de los mapas digitales:

Primero, se calcula el gradiente térmico altitudinal relacionando las diferentes Tma en las estaciones meteorológicas, con la altitud de estas (Domínguez y Justicia, 1993). Esto se consigue aplicando la función regresión lineal que incorpora el software estadístico Minitab y con ello se genera la ecuación 1: $\operatorname{Tm} a=\tau \cdot z+b$

donde Tma es la temperatura mínima absoluta diaria $\left({ }^{\circ} \mathrm{C}\right), \tau$ es el coeficiente de regresión (gradiente térmico altitudinal) y $\mathrm{z}$ es la altitud (msnm).

Segundo, conforme al procedimiento que desarrollan Fries et al. (2012), se emplea una técnica sencilla para corregir el desfase en la altitud de las estaciones y su incidencia en las mediciones de Tma. Para esto, en cada estación se realiza la estimación de Tma a una altitud de referencia $\left(Z_{\text {Det }}\right)$, la cual se estable en 1800 mnsm para minimizar el efecto atmosférico estudiado por Barreto et al. (2009). Por tanto, para estimar Tma se utiliza la ecuación 2:

$$
T m a_{\text {Det }}=T m a_{\text {Obs }}+\left(\tau \cdot\left(Z_{\text {Det }}-Z_{\text {Estación }}\right)\right)
$$

donde $T m a_{D e t}$ es la temperatura mínima absoluta diaria estimada, $T m a_{O b s}$ es la temperatura mínima absoluta diaria observada en la estación, $\tau$ es el gradiente 
altitudinal que se obtiene de la ecuación $1, Z_{D e t}$ es la altitud de referencia y $Z_{\text {Estación }}$ es la altitud de la estación meteorológica.

Tercero, para realizar la predicción de valores en ubicaciones sin medición se hacen interpolaciones. Para ello el software utilizado emplea una fórmula matemática general que forma una suma ponderada de datos como se muestra en la ecuación 3 :

$$
\hat{Z}\left(S_{o}\right)=\sum_{i=1}^{N} \lambda_{i} Z\left(S_{i}\right)
$$

donde $\hat{Z}\left(S_{o}\right)$ es la ubicación de la predicción, $Z\left(\mathrm{~S}_{\mathrm{i}}\right)$ es el valor medio en dicha ubicación, $\lambda_{\mathrm{i}}$ es una ponderación desconocida para el valor medido en la ubicación $i$, $N$ es la cantidad de valores medidos.

Dichas interpolaciones se aplican en métodos geoestadísticos, para lo cual se dispone de herramientas en el software que realizan esto, destacando entre otros el método kriging, el cual tiene diferentes variaciones, entre ellas el kriging ordinario. En este método, la función $y_{y}(h)$ se llama semivariograma y muestra la correlación espacial entre los valores que se representan gráficamente. Las interpolaciones se hacen tanto en el paso cuarto como en el quinto.

Cuarto, para realizar el mapa de temperaturas mínimas en Tenerife se generó previamente el ráster Tma. Esto se hizo empleando la técnica desarrollada por Fries et al. (2012), que consiste en utilizar el DEM para obtener la distribución vertical de Tma en cada pixel (isotermas), aplicando la ecuación 4:

$$
T m a_{(x, y)}=T m a_{D e t}+\left(\tau \cdot\left(Z_{(x, y)}^{D E M}-Z_{D e t}\right)\right)
$$

donde $\operatorname{Tma}_{(x, y)}$ es la temperatura mínima absoluta en un pixel de posición $(x, y)$ en la cuadricula, $T m a_{\text {Det }}$ y $Z_{\text {Det }}$ se obtienen de la ecuación $2, \tau$ se obtiene de la ecuación 1, y $Z_{(x, y)}^{D E M}$ es el DEM.

Por último, se introduce esta ecuación en la herramienta calculadora ráster del software y se genera el ráster Tma.

Quinto, para realizar el mapa de áreas potenciales para el cultivo de los frutales estudiados, se sigue un procedimiento que consiste en: primero se genera un modelo de la pendiente digital en porcentaje a partir del DEM, empleando herramientas SIG de análisis de superficie (Sánchez et al. 1998) y luego se reclasifica en rangos. Segundo, se reclasifica también el ráster de Tma en rangos y se aplican herramientas SIG de álgebra de mapas que incluye un conjunto de operadores aritméticos y lógicos para producir una capa ráster de salida. En este paso, se toma en cuenta la temperatura umbral, siendo para el mango $-3^{\circ} \mathrm{C}$, en guayaba $-2^{\circ} \mathrm{C}$ y en noni $12^{\circ} \mathrm{C}$ (FranciscoBethencourt, 2015) y la pendiente más favorable del terreno fijándose en este caso un rango de 0 a $25 \%$. La temperatura umbral se refiere a la temperatura ambiente más baja que no produce daños a la planta en 30 minutos de exposición. Tercero, se utiliza el análisis de superposición para materializar todos los atributos de las entidades que forman parte de la superposición (Martínez et al. 2000). En este paso se descartan aquellas áreas que de acuerdo con la Ley de Espacios Naturales de Canarias, resultan no conforme con la explotación agrícola y al final quedan delimitadas las áreas potenciales. 


\section{Resultados y discusión}

\subsection{Datos climatológicos}

La distribución en la isla de las estaciones meteorológicas que conforman la red del Cabildo Insular de Tenerife, se puede observar en la Figura 2. En la misma, se presentan parámetros estadísticas como la media $(\overline{\mathrm{X}})$ y desviación estándar (s), de las variables altitud $(\mathrm{H})$ y temperatura mínima absoluta diaria (Tma). Al respecto, el número de observaciones (n) nos indica que hay un equilibrio en cuanto a la distribución de las estaciones entre la vertiente norte y sur de la isla. Por otra parte, indicar que la estación ubicada a mayor altitud se encuentra en el sur (2071 msnm) e igualmente allí se registra la Tma más baja $\left(-2,6^{\circ} \mathrm{C}\right)$.

Figura 2. Distribución espacial de las estaciones meteorológicas.

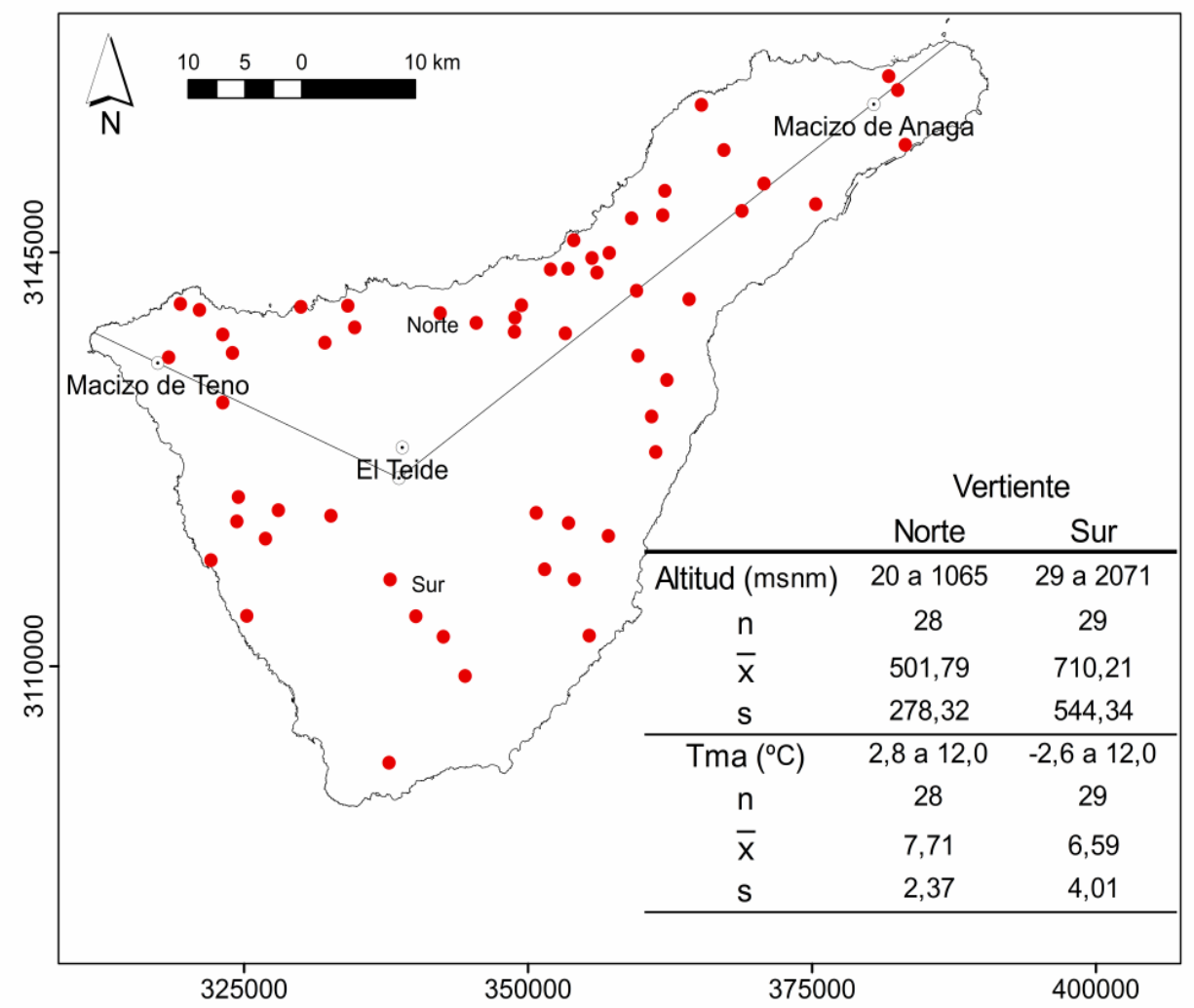

Fuente: Elaboración propia. 


\subsection{Modelo de elevación digital}

El tratamiento de los datos topográficos (curvas de nivel) mediante el SIG nos ha permitido elaborar un DEM de la isla con resolución de $25 \mathrm{~m}$ x $25 \mathrm{~m}$ por pixel (Figura 3).

Figura 3. Modelo de Elevación Digital de la isla de Tenerife.

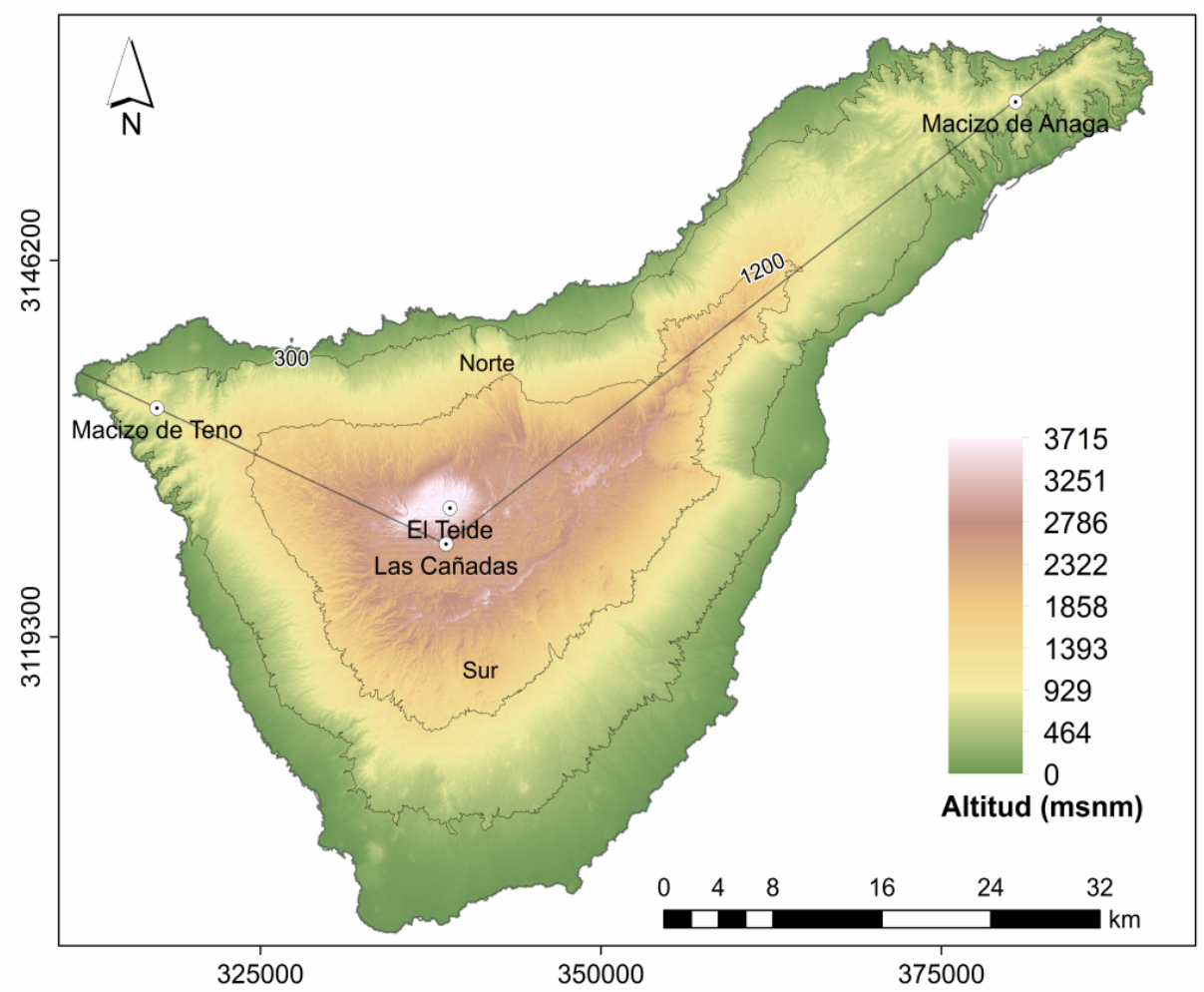

Fuente: Elaboración propia.

\subsection{Gradiente térmico altitudinal}

En el análisis de regresión simple se observaron diferencias significativas $(\mathrm{P} \leq 0,01)$ entre las variables altitud $(\mathrm{H})$ y Tma. Expresando estos resultados a través del coeficiente de determinación $\left(\mathrm{R}^{2}\right)$, indica que el modelo explica el $91,5 \%$ de la variabilidad (Figura 1a). El coeficiente $\mathrm{R}^{2}$ es un buen indicador para medir la fiabilidad de los datos en un modelo de regresión (Leal-Nares et al., 2010). Por otra parte, el gradiente térmico altitudinal $(\tau)$ que se obtiene es $-0,007179\left({ }^{\circ} \mathrm{C} \mathrm{m}{ }^{-1}\right)$, que 
significa los grados que desciende la temperatura por cada metro de altitud (Domínguez y Justicia, 1993; Fries et al., 2012).

\subsection{Confiabilidad del ráster Tma}

El gradiente térmico altitudinal es un dato importante para desarrollar la ecuación 4, a partir de la cual se obtiene el ráster Tma. Para determinar la confiabilidad de dicho ráster se compararon los datos reales observados en las estaciones climatológicas con los datos estimados en las mismas estaciones, pero obtenidos a través del ráster. Se emplearon dos métodos estadísticos: el análisis de correlación para obtener el coeficiente de Pearson (R) y la Raíz del Error Cuadrático Medio (RMSE). Para determinar RMSE se emplea la ecuación 5 (Loague and Green, 1991):

$$
R M S E=\sqrt{\frac{\sum_{i=1}^{n}(P i-O i)^{2}}{n}} \cdot \frac{100}{M}
$$

donde $n$ es el número de observaciones, $P_{i}$ son los valores estimados, $\mathrm{O}_{\mathrm{i}}$ son $\operatorname{los}$ valores observados y $M$ es la media de los valores observados.

El coeficiente de correlación de Pearson que se obtuvo fue 0,949758 lo que indica una correlación muy alta entre los valores observados y los estimados mediante el ráster Tma. Por otra parte, de acuerdo con la $\operatorname{RMSE}(13,54 \%)$ la simulación de los valores de Tma por medio del ráster se considera buena (Figura 1b).

Figura 1. Representación gráfica del análisis estadístico de regresión simple (a) y correlación (b).
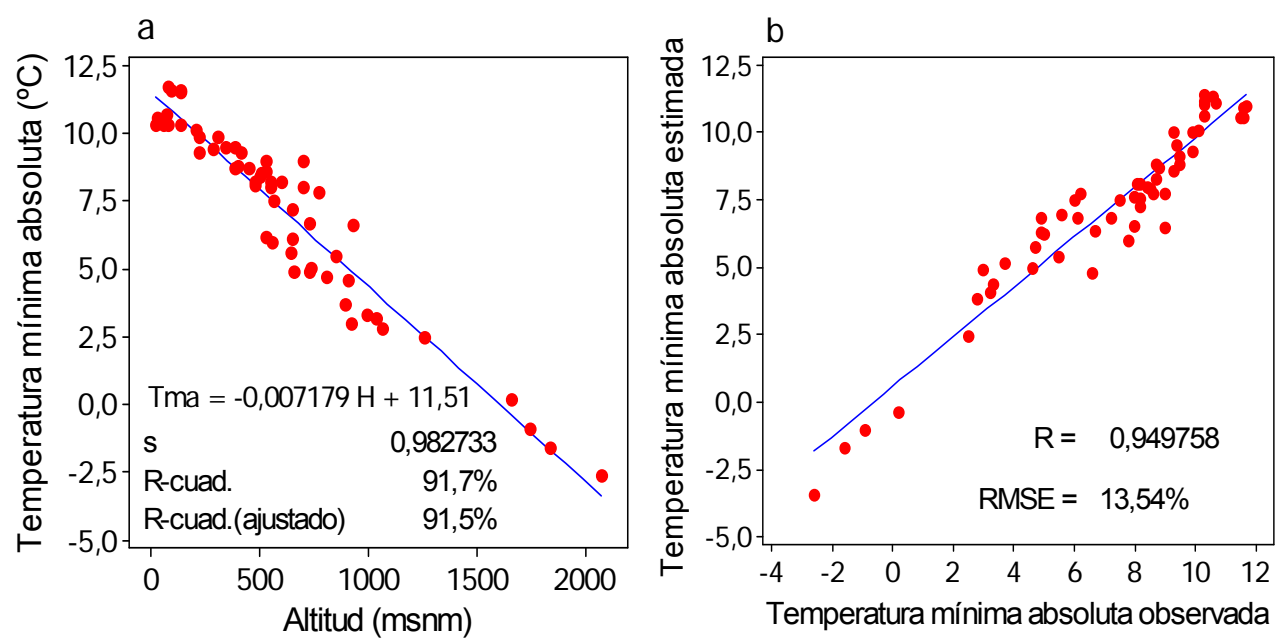

Fuente: Elaboración propia. 


\subsection{Mapa de distribución espacial de bajas temperaturas}

En la Figura 4 se presenta el mapa de distribución espacial de bajas temperaturas, el cual se obtiene a partir del ráster Tma. La distribución por rangos de altitud y temperatura, se ha realizado por medio del siguiente procedimiento empleando herramientas SIG: a) se divide la superficie de la isla $2034 \mathrm{~km}^{2}$ entre el área de cada pixel del ráster $0,000625 \mathrm{~km}^{2}$ y se obtienen 3254400 entidades; estas se convierten luego en un shapefile de puntos (misma cantidad), b) en la tabla de atributos del shapefile se realiza una selección por atributos, ejemplo: de 0 a $200 \mathrm{~m}$ de altitud y se obtiene como resultado los valores de Tma en ese rango de altitud, c) se repite de nuevo el procedimiento para los rangos que faltan.

Figura 4. Mapa de distribución espacial de bajas temperaturas en la isla de Tenerife.

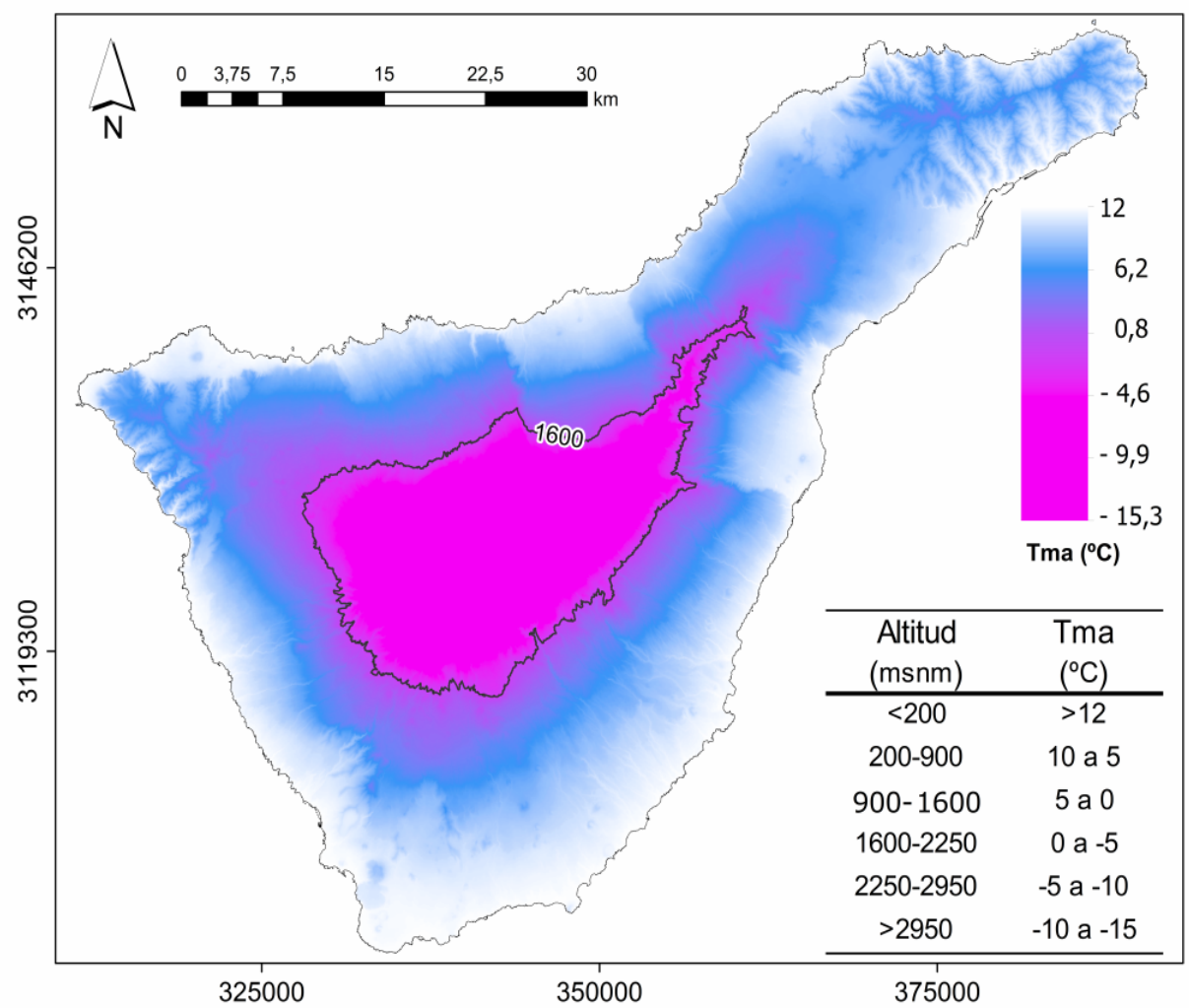

Fuente: Elaboración propia. 
El resultado se puede observar en la tabla que acompaña la leyenda de la Figura 4. Al reagrupar esos datos de acuerdo al criterio de bajas temperaturas se obtiene lo siguiente: 1 ) riesgo por heladas $\left(\mathrm{Tma}<0^{\circ} \mathrm{C}\right.$ ) a una altitud $>1600 \mathrm{msnm}, 2$ ) riesgo por frío (Tma de 0 a $15^{\circ} \mathrm{C}$ ) a una altitud $<1600 \mathrm{msnm}$. De acuerdo con esto, es necesario tomar en cuenta los riesgos climáticos (temperaturas) al momento de emprender la planificación de una explotación agrícola con árboles frutales tropicales y subtropicales, coincidiendo con Rodrigo et al. (2014).

En el caso de una explotación agrícola que se inicia con árboles jóvenes, durante el tiempo que transcurre desde que se plantan hasta su primera floración (que es generalmente a los 3 años), el riesgo de daños tanto por frío como por heladas es un factor muy importante y se debe tomar en cuenta al momento de seleccionar su ubicación.

En atención a los resultados que se observan en la Figura 4, el noni no debería cultivarse a una altitud superior a $200 \mathrm{msnm}$ en la vertiente norte y $300 \mathrm{msnm}$ en la vertiente sur. Este frutal es muy sensible y temperaturas inferiores a $12^{\circ} \mathrm{C} 1 \mathrm{e}$ ocasionan daños por frío (Francisco-Bethencourt, 2015).

Para el mango, Rodríguez y Méndez (2010) fijan una altitud límite de $350 \mathrm{msnm}$ en el norte y $500 \mathrm{msnm}$ en el sur para plantaciones en la isla. Sin embargo en el trópico estos árboles llegan a cultivarse a una altitud superior a $1500 \mathrm{msnm}$ (Galán, 1993). Por otra parte, al comparar la distribución de bajas temperaturas en Tenerife con el hecho de que estos árboles llegan a soportar heladas (hasta una temperatura umbral), resulta factible desarrollar nuevas plantaciones hasta una altitud límite de $1200 \mathrm{msnm}$ en lugares donde la temperatura media anual se encuentre en el rango recomendado por Galán (1993).

La guayaba en la isla, de acuerdo con Fernández y Hernández (2013), se puede cultivar hasta los $800 \mathrm{msnm}$. Por el contrario, en el trópico su cultivo llega inclusive a 1500 msnm (Padilla et al., 2012).

Para soportar heladas los árboles jóvenes de guayaba deben someterse a una aclimatación al frío, lo cual se puede hacer en el vivero sometiéndoles a bajas temperaturas antes de ser trasplantados a campo (Hao et al., 2009). Otra opción si previamente no se hace la aclimatación, es que estos árboles después de ser trasplantados pasen su primer invierno a temperaturas de frío $\left(0\right.$ a $\left.15^{\circ} \mathrm{C}\right)$, sin que estas lleguen a prolongarse por más de 2 horas consecutivas.

Sobre la base de las consideraciones anteriores, es factible desarrollar nuevas plantaciones de guayaba hasta una altitud límite de $1200 \mathrm{msnm}$ en lugares donde la temperatura media anual alcance el rango recomendado por Fernández y Hernández (2013) y, los árboles jóvenes sean aclimatados.

En consecuencia, es importante tomar en cuenta el mapa elaborado de distribución de bajas temperaturas en la isla. Por otra parte, el riesgo de bajas temperaturas también ha sido cartografiado por otros autores, que además reportan la relación que existe entre este y el rendimiento de frutales subtropicales. En este sentido, Kordi et al. (2015) presentan un mapa de riesgo y reportan que los años con más días de heladas reducen drásticamente el rendimiento de la granada y la higuera. 


\subsection{Mapa de áreas potenciales para nuevas plantaciones}

Para que la cartografía elaborada tenga una representación suficientemente clara y para que su utilización pueda tener carácter práctico en la planificación de nuevas plantaciones de los cultivos estudiados, resulta conveniente establecer zonas o áreas de potencialidad, en función de los valores alcanzados con los tres indicadores evaluados: temperatura mínima absoluta diaria (Tma), temperatura media anual (Tmed) y altitud. La distribución territorial de las áreas potenciales para el cultivo de mango, guayaba y noni en Tenerife, ha sido generada desde el punto de vista fisiográfico (altitud y pendiente) y climático (temperatura Tma y Tmed). En este caso la temperatura se estima de acuerdo con un comportamiento térmico normal.

Finalmente se han clasificado dos áreas potenciales. La descripción de dichas áreas es como sigue: la zona 1 (verde), es el área adecuada para los tres cultivos (mango, guayaba y noni). Esta zona delimita la altitud máxima para cultivar el noni. La zona 2 (amarillo), es adecuada para el cultivo de mango y guayaba y, delimita la altitud máxima en el caso del mango. Para plantaciones de guayaba a una altitud superior a $300 \mathrm{msnm}$ (hasta $1200 \mathrm{msnm}$ ), se debe considerar la aclimatación de los árboles jóvenes.

En cuanto a estos resultados, la cartografía elaborada muestra la importancia que tiene en una superficie agrícola, la distribución espacial de la temperatura. En este caso, en la tabla que acompaña la Figura 5 resulta evidente la diferencia entre las dos zonas en cuanto a Tma, al contrario de Tmed que se encuentra en un rango equilibrado. Por otra parte destacar que en Tenerife en un año con comportamiento término normal, las temperaturas absolutas en los meses de invierno a una altitud $<1600 \mathrm{msnm}$ son superiores a $0^{\circ} \mathrm{C}$.

Recientemente, otros estudios han estudiado y cartografiado también el riesgo de bajas temperaturas en cultivos frutales, lo que denota la preocupación por conocer sus efectos. En la Región de Murcia (España), Pérez (2016) presenta un mapa de exposición al riesgo de heladas para viña y cítricos, entre otros. La conclusión de este estudio es que en los meses de enero y febrero suceden las mayores pérdidas por dicho factor. Por otra parte en Asia Occidental, Zabihi et al. (2016) realizan un estudio de zonas agroecológicas adecuadas para el cultivo de cítricos, tomando en cuenta para ello el riesgo de bajas temperaturas (frío y heladas). En este estudio determinan la temperatura mínima y la altitud que tiene un impacto significativo en la distribución espacial de los cítricos.

A su vez, en la planificación de una explotación agrícola de frutales tropicales y subtropicales tiene especial relevancia los trabajos cartográficos en base a la estimación de la temperatura, por la repercusión que evidencian los estudios sobre bases fisiológicas del crecimiento de dichos frutales. Al respecto, la temperatura es importante para la estimulación de la floración de árboles como el mango y cambios abruptos en la temperatura nocturna pueden causar una floración asíncrona (Hedhly, 2011). Por otra parte, Dinesh y Reddy (2012) señalan que si el invierno es muy frío y 
le sigue un aumento de la temperatura diurna a medida que se aproxima el verano, el resultado es una pobre floración.

Figura 5. Mapa de áreas potenciales para el cultivo de mango, guayaba y noni en la isla de Tenerife.

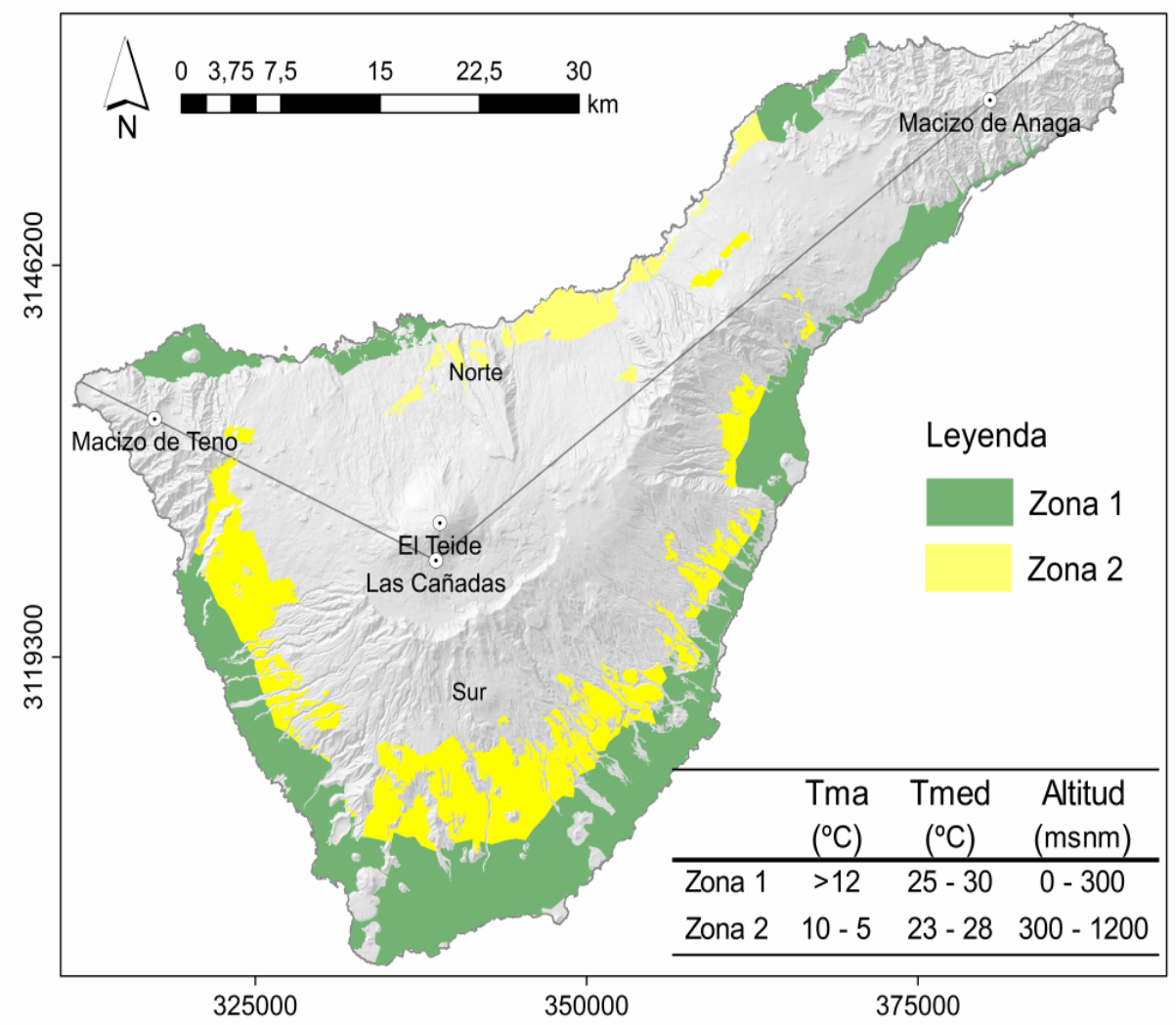

Fuente: Elaboración propia.

El poder seleccionar el área potencial para la plantación de un árbol frutal, es compatible con el propósito que se persigue de este estudio, que es el manejo del cultivo en agricultura ecológica dado que las condiciones climáticas (temperatura) influyen en su desarrollo. Este manejo deseable, no es la única opción que tiene el agricultor. Se debe acotar también que para el crecimiento y desarrollo del cultivo, tiene que proveerse a los árboles de una adecuada fertilización, contar con disponibilidad de agua para riego, tener el suelo un buen drenaje, entre otros aspectos que no son objetivo de esta investigación. 


\section{Conclusiones}

El método utilizado para obtener el ráster de temperaturas mínimas absolutas diarias, ha permitido realizar una buena simulación de la distribución de bajas temperaturas (frío y heladas) en la isla de Tenerife. Esta información se ha empleado para preparar un mapa digital y extraer de este, datos para delimitar rangos de temperatura en función de rangos de altitud. La combinación de capas gráficas a partir de datos ráster permitió elaborar la cartografía con una inversión mínima de recursos.

En el mapa se han delineado áreas potenciales para el cultivo de mango, guayaba y noni. Esto constituye una herramienta útil para la planificación presente y futura de nuevas explotaciones agrícolas donde el manejo del cultivo puede orientarse hacia una agricultura ecológica, aunque ello no es exclusivo. Por otra parte, el mapa al estar en formato SIG puede ser incorporado a aplicaciones informáticas más complejas.

Se recomienda realizar nuevos estudios para evaluar con otros frutales tropicales y subtropicales las áreas potenciales para su cultivo en la isla, debido a que pueden sufrir también daños por bajas temperaturas.

\section{Bibliografía}

Barreto, A., Arbelo, M., Nuñez-Casillas, L., Hernández-Leal, P., González-Calvo, A. y Alonso-Benito, A. (2009): Estimación de la emisividad espectral con datos térmicos aster de la isla de Tenerife. En Montesinos, S. y Fernández, L. (Eds.): Teledetección: Agua y desarrollo sostenible. Barcelona, XIII congreso de la Asociación Española de Teledetección, 525-528. Disponible en: http://www.aet.org.es/congresos/xiii/cal132.pdf

Cardós, C., Barrera, E. y Sanz, R. (2006): Un estudio sobre episodios de temperaturas extremas en Canarias. En Instituto Nacional de Meteorología (Ed.): Calendario Meteorológico 2007. Madrid, Ministerio de Medio Ambiente, 239-248.

Castillo, J. (1999): Factores climáticos de la agricultura ecológica en el Almanzora. Nimbus, 3, 63-91.

Dinesh, M.R. y Reddy, B.M. (2012): Physiological basis of growth and fruit yield characteristics of tropical and sub-tropical fruits to temperature. In Sthapit, B., Ramanatha, V. y Sthapit, S. (Eds.): Tropical fruit tree species and climate change. New Delhi, Biodiversity International publishers, 45-70.

Domínguez, R. y Justicia, A. (1993): Gradientes térmicos en Andalucía. Baetica: Estudios de arte, geografía e historia, 15, 65-97.

Dorta, P. (2007): Catálogo de riesgos climáticos en Canarias: Amenazas y vulnerabilidad. Geographicalia, 51, 133-160.

Easterling, D.R., Kunkel, K.E., Wehner, M.F. y Sun, L. (2016): Detection and attribution of climate extremes in the observed record. Weather and Climate Extremes, 11, 17-27.

Fernández, D. y Hernández, P. (2013): El guayabo. Tenerife, Instituto Canario de Investigaciones Agrarias. 
Francisco-Bethencourt, D.A. (2015): Bajas temperaturas y frutales leñosos tropicales y subtropicales en Canarias: Consideraciones en el transporte de plantones. Sevilla, Editorial Escritura entre las nubes.

Fries, A., Rollenbeck, R., Nauss, T., Peters, T. y Bendix, J. (2000): Near surface air humidity in a megadiverse Andean mountain ecosystem of Southern Ecuador and its regionalization. Agricultural and Forest Meteorology, 152, 17-30.

Galán, S. (1993): The situation of mango culture in the world. Acta Horticulturae, 341, 31-38.

Hao, W., Arora, R., Yadav, A.K. y Joshee, N. (2009): Freezing tolerance and cold acclimation in guava (Psidium guajava L.). HortScience, 44, 1258-1266.

Hedhly, A. (2011): Sensitivity of flowering plant gametophytes to temperature fluctuations. Environmental and Experimental Botany, 74, 9-16.

Henríquez, C., Méndez, J. y Masis, R. (2013): Interpolación de variables de fertilidad de suelos mediante el análisis kriging y su validación. Agronomía Costarricense, 37, 71-82.

Izquierdo, T. y Márquez, A. (2007): Estudio comparativo de diferentes métodos de interpolación para la realización de mapas de precipitación en la isla de la Gomera (Islas Canarias): Evaluación de la incorporación de la altitud. Geogaceta, 42, 131-134.

Kordi, B., Khosravi, M. y Mahmoodi, P. (2015): Mapping and analyzing the risk of frost and chilling damage on horticultural crops (study case: Taftan border area, Southeast of Iran). Journal of Biodiversity and Environmental Sciences, 7(2), 261-275.

Leal-Nares, O., Mendoza, M. y Carranza, E. (2010): Análisis y modelamiento espacial de información climática en la cuenca de Cuitzeo, México. Investigaciones Geográficas, 72, 49-67.

Loague, K. y Green, R. (1991): Statistical and graphical methods for evaluating solute transport models: overview and application. Journal of Contaminant Hydrology, 7, 51-73.

Lopéz-Manzanes, F. y Machín, N. (2012): Los cultivos de Tenerife: Aspectos territoriales. Tenerife, Cabildo Insular de Tenerife.

MAGRAMA. (2013): Agricultura ecológica. Estadísticas 2012. Madrid, Ministerio de Agricultura, Alimentación y Medio Ambiente.

MAGRAMA. (2014): Agricultura ecológica. Estadísticas 2013. Madrid, Ministerio de Agricultura, Alimentación y Medio Ambiente.

Martínez, V., Dal-Ré, R., García, A. y Ayuga, F. (2000): Modelación distribuida de la escorrentía superficial en pequeñas cuencas mediante SIG: Evaluación experimental. Ingeniería Civil, 104, 141-146.

Nelson, S. (2003): Morinda citrifolia L. Rubiaceae (Rubioideae) Coffee family. Hawaii, Permanent Agriculture Resources (PAR) publishers.

Padilla, R., González, G., Pérez, B., Osuna, G., Espinola, B. y Reyes, A. (2012): Phenological behavior of guava trees (Psidium guajava L) under different climatic conditions of México. Acta Horticulturae, 959, 97-102.

Paul, V., Pandey, R. y Singh, A. (2011): Vulnerability of trees and fruit crops to climate change. In Pal, M., Khetarpal, S., Pandey, R. y Kumar, P. (Eds): Climate change: Impacts and adaptations in crop plants. New Delhi, Today and Tomorrow`s publishers, 169-186.

Pérez, A. (2016): Riesgos de heladas en cultivos: Análisis y evaluación. En Vera, J.F., Olcina, J. y Hernández, M. (Eds.): Paisaje, cultura territorial y vivencia de la geografía: Libro homenaje al profesor Alfredo Morales Gil. Alicante, Universidad de Alicante, 1005-1022. 
Rodrigo, J., Senciales, J. y González, J. (2004): La necesidad de considerar los riesgos climáticos en la introducción de cultivos tropicales en latitudes medias: El mango en el valle del Guadalhorce (Málaga). Investigaciones Geográficas, 62, 127-141.

Rodríguez, L. y Méndez, C. (2010): Manejo de plantaciones nuevas de Mango. Tenerife, Cabildo Insular de Tenerife.

Sánchez, F., Tejero, R. y Bergamín, J. (1998): Análisis de la variabilidad del relieve a partir de modelos digitales del terreno. Revista de la Sociedad Geológica de España, 11, 139-149.

Zabihi, H., Vogeler, I., Amin, Z.M. y Gourabi, B.R. (2016): Mapping the sensitivity of citrus crops to freeze stress using a geographical information system in Ramsar, Iran. Weather and climate extremes, 14, 17-23. 